\title{
Inferences for Joint Hybrid Progressive Censored Exponential Lifetimes under Competing Risk Model
}

\author{
Abduallah M. Almarashi, ${ }^{1}$ Ali Algarni, ${ }^{1}$ A. M. Daghistani, ${ }^{1}$ G. A. Abd-Elmougod, ${ }^{2}$ \\ S. Abdel-Khalek, ${ }^{3,4}$ and Mohammad Z. Raqab $\mathbb{D}^{\mathbf{5}}$ \\ ${ }^{1}$ Statistics Department, Faculty of Science, King Abdulaziz University, Jeddah, Saudi Arabia \\ ${ }^{2}$ Mathematics Department, Damanhour University, Damanhour, Egypt \\ ${ }^{3}$ Department of Mathematics and Statistics, College of Science, Taif University, PO Box 11099, Taif 21944, Saudi Arabia \\ ${ }^{4}$ Mathematics Department, Sohag University, Sohag, Egypt \\ ${ }^{5}$ Mathematics Department, The University of Jordan, Amman 11942, Jordan \\ Correspondence should be addressed to Mohammad Z. Raqab; mraqab@ju.edu.jo
}

Received 2 July 2021; Accepted 13 August 2021; Published 25 August 2021

Academic Editor: Ibrahim Almanjahie

Copyright (C) 2021 Abduallah M. Almarashi et al. This is an open access article distributed under the Creative Commons Attribution License, which permits unrestricted use, distribution, and reproduction in any medium, provided the original work is properly cited.

\begin{abstract}
The aim of this paper is devoted to the problem of comparative life tests under joint censoring samples from an exponential distribution with competing risks model. This problem is considered under the consideration that only two causes of failure are occurring and the units come from two production lines such that the exponential failure time of units is censored under a hybrid progressive Type-I censoring scheme. Maximum likelihood estimation and different Bayes methods of estimation are discussed. The asymptotic confidence intervals as well as the Bayes credible intervals are established. A real data set representing time to failure on two groups of strain male mice receiving radiation is analyzed for illustrative purposes. All theoretical results are assessed and compared through the Monte Carlo study.
\end{abstract}

\section{Introduction}

Under the joint progressive censoring (JPC), the products from two different production lines, say Population- $\eta_{1}$ (Pop$\left.\eta_{1}\right)$ and Population $-\eta_{2}\left(\right.$ Pop $\left.-\eta_{2}\right)$, are combined and put on a life testing experiment. Then, comparative life testing is done on these products to measure the relative merits of two competing durations of life products. Suppose that the units are produced from two different lines (Pop- $\eta_{1}$ and Pop- $\eta_{2}$ ) under the same conditions. Then, the experimenter can randomly select the two independent samples to put under time testing experiment. Due to the time and cost constraints, the termination of the statistical experiment arises naturally in various fields such as reliability, survival, and medical studies. For a detailed study of this type of data and related inferences, one may refer to Rao et al. [1], Basu [2], Johnson and Mehrotra [3], Mehrotra and Johnson [4], Bhattacharyya and Mehrotra [5], and Mehrotra and
Bhattacharyya [6]. Under the JPC scheme, the two samples from Pop $-\eta_{1}$ and Pop- $\eta_{2}$ of sizes $m$ and $n$, respectively, are combined and put on a life testing experiment. Balakrishnan and Rasouli [7] and Rasouli and Balakrishnan [8] introduced the JPC scheme when the data can be produced from two exponential populations. Shafay et al. [9] discussed the Bayesian inference based on a jointly Type-II censored sample from two exponential populations. Mondal and Kundu [10] addressed the problem of point and interval estimation of the unknown parameters of two Weibull distributions based on the Bayesian approach.

In jointly Type-II censoring scheme, suppose that a sample of size $D$ is taken from the first production line, Pop$\eta_{1}$, with cumulative distribution function (CDF) $F_{1}$ and probability density function (PDF) $f_{1}$, say, $\left\{X_{1}, X_{2}, \ldots, X_{D}\right\}$. Also, another independent sample of size $N$ is taken from the second production line, Pop- $\eta_{2}$, with CDF $F_{2}$ and PDF $f_{2}$, say, $\left\{Y_{1}, \ldots, Y_{N}\right\}$. Let $m$ be the total 
number of observed failures in the experiment. Then, the ordered lifetime sample $\left(T_{1}, T_{2}, \ldots, T_{m}\right)$ forms a combined sample taken from both samples $\left\{X_{1}, X_{2}, \ldots, X_{D_{m}}\right\}$ and $\left\{Y_{1}, Y_{2}, \ldots, Y_{N_{m}}\right\}$ with $m=D_{m}+N_{m}$ being the joint Type-II censoring sample. Hence, the observed data consists of $(\mathbf{T}, \omega)=\left\{\left(T_{1}, \omega_{1}\right),\left(T_{2}, \omega_{2}\right), \ldots,\left(T_{m}, \omega_{m}\right)\right\}$,

$$
L(\mathbf{T}, \boldsymbol{\omega})=\frac{D ! N !}{\left(D-n_{1}\right) !\left(N-n_{2}\right) !}\left(\prod_{i=1}^{m}\left[f_{1}\left(t_{i}\right)\right]^{\omega_{i}}\left[f_{2}\left(t_{i}\right)\right]^{1-\omega_{i}}\right)\left[S_{1}\left(t_{m}\right)\right]^{D-n_{1}}\left[S_{2}\left(t_{m}\right)\right]^{N-n_{2}}
$$

where $S_{1}($.$) and S_{2}($.$) are the reliability functions of X$ and $Y$, respectively.

In the conventional Type-I censoring scheme, the experiment continues up to the prespecified time $\tau$. On the other hand, the conventional Type-II censoring scheme requires the experiment to continue until a prespecified number of failures occur. If a mixture of Type-I and Type-II censoring schemes is applied, then the resulting censoring scheme is called a hybrid censoring scheme (HCS). In Type-I HCS, the test is terminated when a prefixed number, $m<D+N$, out of $D+N$ items has failed, or when a prefixed time, $\tau$, has been reached. In other words, the life test is terminated at a random time $T^{*}=\min \left(T_{m}, \tau\right)$. In Type-II HCS, the experiment is stopped when $T^{*}=\max \left(T_{m}, \tau\right)$ is reached.

Moreover, the progressive censoring scheme can be adopted combined with a hybrid scheme to define Type-I hybrid progressive censoring scheme (Type-I HPCS), and it is described as follows. Suppose that a sample of size $n$ is randomly selected from the products to put under test and given an integer $m$, ideal test time $\tau$, and censoring scheme $\mathbf{R}=\left(R_{1}, R_{2}, \ldots, R_{m}\right)$ such that $n=m+\sum_{i=1}^{m} R_{i}$ are determined. At each failure time $T_{i}, R_{i}$ survival units are removed from the test, where $i=1,2, \ldots, J$ and $1 \leq J \leq m$. The experiment is terminated under Type-I HPCS at $T^{*}$, which is defined by $T^{*}=\min \left(T_{m}, \tau\right)$. In this work, we focus our attention on Type-I HPCS. The HCS is quite useful in the reliability acceptance test. See, for example, Childs et al. [11] and Jeong et al. [12] for some development of the hybrid censored sampling plan for the exponential lifetime distributions.
$1 \leq m \leq D+N$ with $\omega_{i}=1$ or 0 accordingly as $T_{i}$ is taken from an $X$-or $Y$-sample, respectively. Let us denote the number of failed units from Pop- $\eta_{1}$ and Pop- $\eta_{2}$ by $n_{1}=$ $\sum_{i=1}^{m} \omega_{i}$ and $n_{2}=m-n_{1}=\sum_{i=1}^{m}\left(1-\omega_{i}\right)$, respectively. Then, the likelihood function based on a JPC sample is given by
In real-life testing (medical and survival studies), it is quite common that more than one risk factor may be present at the same time, and only the smallest failure time can be observed when multiple modes of failure work together. An investigator is often interested in the assessment of specific risk in the presence of other risk factors. For this reason, the observed data consist of a failure time and an indicator denoting the cause of failure, and this model is known as the competing risks model. This aspect is discussed early by Cox [13] using the exponential data. Different properties of the competing risks model are exposed by Crowder [14], Balakrishnan [15], and Bakoban and Abd-Elmougod [16]. Recently, the properties of a competing risks model under the accelerated life test model are discussed by Ganguly and Kundu [17] and Algarni et al. [18]. In a random sample of size $n$ of the life products and predetermined integer munder the competing risks model, the two causes of failure are independent. If $\delta_{i}$ denotes the cause of failure of the $i$-th ordered unit, then in this particular case, $\delta_{i}$ can take only two values $\left(\delta_{i}=k, k=1,2\right)$. Suppose that, for given $n$ and $m$, the failure time and the corresponding cause of failure are recorded; say $\left\{\left(T_{i}, \delta_{i}\right), i=1,2, \ldots, m\right\}$ is observed. For a given censoring scheme $\mathbf{R}=\left(R_{1}, R_{2}, \ldots, R_{m}\right)$, the final censored competing risk sample is abbreviated as

$$
(\mathbf{t}, \boldsymbol{\delta}, \mathbf{R})=\left\{\left(t_{1}, \delta_{1}, R_{1}\right),\left(t_{2}, \delta_{2}, R_{2}\right), \ldots,\left(t_{m}, \delta_{m}, R_{m}\right)\right\} .
$$

Then, the likelihood function can be expressed as

$$
L(\mathbf{t}, \boldsymbol{\delta}, \mathbf{R})=\frac{n !\left(S_{1}\left(t_{m}\right) S_{2}\left(t_{m}\right)\right)^{(n-m)}}{(n-m) !}\left[\prod_{i=1}^{m}\left[f_{1}\left(t_{i}\right) S_{2}\left(t_{i}\right)\right]^{\rho\left(\delta_{i}=1\right)}\left[f_{2}\left(t_{i}\right) S_{1}\left(t_{i}\right)\right]^{\rho\left(\delta_{i}=2\right)}\left(S_{1}\left(t_{i}\right) S_{2}\left(t_{i}\right)\right)^{R_{i}}\right]
$$

where

$$
\rho\left(\delta_{i}=k\right)= \begin{cases}1, & \delta_{i}=k \\ 0, & \delta_{i} \neq k\end{cases}
$$

with $k=1,2$ and $0<t_{1}<t_{2}<\cdots<t_{m}<\infty$.
The aim of this paper is to provide an analysis of Type-I HPC competing risks data. In latent failure time modeling, it is assumed that competing causes of failures are independent random variables. In this paper, it is assumed that the lifetimes of the competing causes of failure follow an exponential distribution with different scale parameters. The 
frequentist and Bayesian methods are applied for estimating the parameters involved in the underlying model. Because of the very complicated nature of the distribution functions, we propose the asymptotic confidence intervals (CIs) and two Bayes credible intervals (CrIs). The paper is summarized as follows. In Section 2, the model assumptions and descriptions are presented. The maximum likelihood estimators (MLEs) and the corresponding CIs are developed in Section 3. Also, point and interval estimation using the Bayes approach is discussed in Section 4. In Section 5, we analyze a real data set for illustrative purposes and perform a numerical simulation study for assessing the so-developed methods. Finally, our findings and brief comments are summarized in Section 6.

\section{The Model Description}

Suppose that the joint sample of size $(n=N+D)$ of units is randomly selected from the two different production lines (Pop- $\eta_{1}$, Pop- $\left.\eta_{2}\right)$ under the same facility. The sample of size $D$ is selected from the first production line (Pop- $\left.\eta_{1}\right)$, and another independent sample of size $N$ is selected from the second production line $\left(\operatorname{Pop}-\eta_{2}\right)$. Suppose that integer $m$ denotes the number of failures needed for statistical inferences, $\tau$ is the ideal test time, and the experiment is running under a censoring scheme $\mathbf{R}=\left(R_{1}, R_{2}, \ldots, R_{m}\right)$. When the first failure $T_{1}$ is observed, its corresponding type $\omega_{1}$ (line- $\eta_{1}$ or line- $\eta_{2}$ ) and failure cause $\delta_{1}$ (first or second cause of failure) are observed; say $\left(T_{1}, \omega_{1}, \delta_{1}\right)$ is recorded. At the second failure $T_{2}$, we also observe $\omega_{2}$ and $\delta_{2}$, and then, $\left(T_{2}, \omega_{2}, \delta_{2}\right)$ is recorded. The experiment continues until the $\min \left(\tau, T_{m}\right)$ is observed. Under this censoring scheme, we have one of the following two types of observations:

$$
\begin{aligned}
& \text { Case I: }\left\{t_{1}<t_{2}<\cdots<t_{m}\right\}, \\
& \text { Case II: }\left\{t_{1}<t_{2}<\cdots<t_{J}\right\}, \quad \text { for } 1 \leq J<m,
\end{aligned}
$$

where $J$ denotes the number of failures observed before time $\tau$. The experiment is terminated at $\tau$ if $\tau<T_{m}$ and terminated at $T_{m}$ if $\tau>T_{m}$. Note that, in Case I, we may consider $J=m$, and for Case II, $1 \leq J<m$. Therefore, the joint Type-I HPCS competing risks sample is

$$
(\mathbf{t}, \boldsymbol{\omega}, \boldsymbol{\delta})=\left\{\left(t_{1}, \omega_{1}, \delta_{1}\right),\left(t_{2}, \omega_{2}, \delta_{2}\right), \ldots,\left(t_{J}, \omega_{J}, \delta_{J}\right)\right\}, \quad 1 \leq J \leq m .
$$

Let the number of units failed from Pop- $\eta_{1}$ be denoted by $m_{1}=\sum_{i=1}^{J} \omega_{i}$, and let the number of units failed from Pop$\eta_{2}$ be $m_{2}=\sum_{i=1}^{J}\left(1-\omega_{i}\right)$. For the two causes of failure, $j=$ 1 , 2, we assume that $n_{1 j}=\sum_{i=1}^{m} \omega_{i} * \rho\left(\delta_{i}=j\right)$, the number of failed units coming from the Pop- $\eta_{1}$ with the cause $j$. Also, $n_{2 j}=\sum_{i=1}^{m}\left(1-\omega_{j}\right) * \rho\left(\delta_{i}=j\right)$, the number of failed units coming from Pop $-\eta_{2}$ with the cause $j, j=1,2$. Based on the exponential lifetimes' data, we report the following assumptions:

(1) The failure times $T_{i}, 1,2, \ldots, J$ are defined as $T_{i}=$ $\min \left\{T_{i k 1}, T_{i k 2}\right\}$ with $T_{i k j}$ being the $i$ - th failure time from the $k$-th population $(k=1,2)$ under cause $j(j=1,2)$.

(2) With parameters $\theta_{k j}$, the random variable $T_{i k j}(k=1,2$ and $j=1,2)$ has the exponential distribution with PDF and CDF given, respectively, by

$f_{k j}(t)=\theta_{k j} \exp \left(-\theta_{k j} t\right), \quad t>0, \theta_{k j}>0, k=1,2$ and $j=1,2$,

$F_{k j}(t)=1-\exp \left(-\theta_{k j} t\right)$

The corresponding reliability and hazard rate functions are, respectively, given by

$$
\begin{aligned}
& S_{k j}(t)=\exp \left(-\theta_{k j} t\right), \\
& h_{k j}(t)=\theta_{k j},
\end{aligned}
$$

where $k$ and $j$ denote the type and cause of the failure, respectively.

(3) The latent failure time is distributed with exponential distribution with $\theta_{k 1}+\theta_{k 2}$ scale parameters.

(4) The discrete random variables $n_{k 1}$ and $n_{k 2}$ which describe the number of failed units under the first and second causes of failure are distributed as binomial distributions with sample size $J-m_{k}$ and probability of success $\theta_{k 1} /\left(\theta_{k 1}+\theta_{k 2}\right)$ and $\theta_{k 2} /\left(\theta_{k 1}+\theta_{k 2}\right)$, respectively.

Based on these assumptions, the joint likelihood function of the observed sample $(\mathbf{t}, \omega, \delta)$ is given by

$$
\begin{aligned}
L(\mathbf{t}, \boldsymbol{\omega}, \boldsymbol{\delta} \mid \phi) & \propto \prod_{i=1}^{J}\left[\left(h_{11}\left(t_{i}\right)\right)^{\rho\left(\delta_{i}=1\right)}\left(h_{12}\left(t_{i}\right)\right)^{\rho\left(\delta_{i}=2\right)}\left(S_{11}\left(t_{i}\right) S_{12}\left(t_{i}\right)\right)\right]^{\omega_{i}}\left(S_{11}\left(t_{i}\right) S_{12}\left(t_{i}\right)\right)^{R_{1 i}} \\
& \times\left[\left(h_{21}\left(t_{i}\right)\right)^{\rho\left(\delta_{i}=1\right)}\left(h_{22}\left(t_{i}\right)\right)^{\rho\left(\delta_{i}=2\right)}\left(S_{21}\left(t_{i}\right) S_{22}\left(t_{i}\right)\right)\right]^{1-\omega_{i}}\left(S_{21}\left(t_{i}\right) S_{22}\left(t_{i}\right)\right)^{R_{2 i}} \\
& \times\left[S_{11}\left(t^{*}\right) S_{12}\left(t^{*}\right)\right]^{D-m_{1}}\left[S_{21}\left(t^{*}\right) S_{22}\left(t^{*}\right)\right]^{N-m_{2}},
\end{aligned}
$$


where $R_{1 i}+R_{2 i}=R_{i}$ with $R_{1 i}$ and $R_{2 i}$ being the number of censored items from the two production lines, Pop- $\eta_{1}$ and Pop- $\eta_{2}$, respectively, $\varphi=\left(\theta_{11}, \theta_{12}, \theta_{21}, \theta_{22}\right)$,

$$
\begin{gathered}
t^{*}= \begin{cases}\tau, & \text { if } \tau<T_{m}, \\
T_{m}, & \text { if } \tau>T_{m},\end{cases} \\
0<t_{1}<t_{2}<\cdots<t_{J}<\infty .
\end{gathered}
$$

\section{Maximum Likelihood Estimation}

Here, in this section, we obtain the MLEs of all parameters involved in the previous model based on the times to failure under Type-I HPC with two factors including the failure type and failure cause. Further, the asymptotic CIs for the model parameters are also obtained. The likelihood function based on the exponential failure times with PDF and CDF given in (7) and (8) can be expressed in the following form:

$$
\begin{aligned}
& L\left(\theta_{11}, \theta_{12}, \theta_{21}, \theta_{22} \mid \phi\right) \propto \theta_{11}^{n_{11}} \theta_{12}^{n_{12}} \theta_{21}^{n_{21}} \theta_{22}^{n_{22}} \exp \\
& \left.\quad\left\{\begin{array}{c}
-\left(\theta_{11}+\theta_{12}\right) \sum_{i=1}^{J}\left(R_{1 i}+\omega_{i}\right) t_{i}-\left(D-m_{1}\right)\left(\theta_{11}+\theta_{12}\right) t^{*} \\
-\left(N-m_{2}\right)\left(\theta_{21}+\theta_{22}\right) t^{*}
\end{array}\right)-\left(\theta_{21}+\theta_{22}\right) \sum_{i=1}^{J}\left(R_{2 i}+\left(1-\omega_{i}\right)\right) t_{i}\right\}
\end{aligned}
$$

From (12), we immediately obtain the log-likelihood function as

$$
\begin{aligned}
\ell\left(\theta_{11}, \theta_{12}, \theta_{21}, \theta_{22} \mid \underline{t}\right) \propto & n_{11} \log \theta_{11}+n_{12} \log \theta_{12}+n_{21} \log \theta_{21}+n_{22} \log \theta_{22}-\left(\theta_{11}+\theta_{12}\right) \\
\times & \sum_{i=1}^{J}\left(R_{1 i}+\omega_{i}\right) t_{i}-\left(\theta_{21}+\theta_{22}\right) \sum_{i=1}^{J}\left(R_{2 i}+\left(1-\omega_{i}\right)\right) t_{i} \\
& -\left\{\left(D-m_{1}\right)\left(\theta_{11}+\theta_{12}\right)+\left(N-m_{2}\right)\left(\theta_{21}+\theta_{22}\right)\right\} t^{*}
\end{aligned}
$$

The partial derivatives of the log-likelihood function (13) are reduced to

$$
\frac{\partial \ell\left(\theta_{11}, \theta_{12}, \theta_{21}, \theta_{22} \mid \underline{t}\right)}{\partial \theta_{k j}}=0, \quad k, j=1,2,
$$

which is readily reduced to

$$
\begin{aligned}
& \widehat{\theta}_{1 j}=\frac{n_{1 j}}{\sum_{i=1}^{J}\left(R_{1 i}+\omega_{i}\right) t_{i}+\left(D-m_{1}\right) t^{*}}, \quad j=1,2, \\
& \widehat{\theta}_{2 j}=\frac{n_{2 j}}{\sum_{i=1}^{J}\left(R_{2 i}+\left(1-\omega_{i}\right)\right) t_{i}+\left(N-m_{2}\right) t^{*}}, \quad j=1,2 .
\end{aligned}
$$

Remark 1. Under the setting $n_{1 j}=\sum_{i=1}^{J} \omega_{i} * \rho\left(\delta_{i}=j\right)=0$ or Dand $n_{2 j}=\sum_{i=1}^{m}\left(1-\omega_{i}\right) * \rho\left(\delta_{i}=j\right)=0$ or $N$, equations (15) and (16) do not lead to the existence of the MLEs of $\theta_{1 j}, \theta_{2 j}, j=1,2$. Further, the exact probability distributions of the estimators $\widehat{\theta}_{1 j}$ and $\widehat{\theta}_{2 j}$ are defined as the mixture of discrete and continuous distributions, which are difficult to be derived (see, for example, [19]).

From the joint log-likelihood function given by (12), the second partial derivatives are derived to be

$$
\begin{aligned}
& \frac{\partial^{2} \ell\left(\theta_{11}, \theta_{12}, \theta_{21}, \theta_{22} \mid \underline{t}\right)}{\partial \theta_{1 j}^{2}}=\frac{-n_{1 j}}{\theta_{1 j}^{2}}, \quad j=1,2, \\
& \frac{\partial^{2} \ell\left(\theta_{11}, \theta_{12}, \theta_{21}, \theta_{22} \mid \underline{t}\right)}{\partial \theta_{2 j}^{2}}=\frac{-n_{2 j}}{\theta_{2 j}^{2}}, \quad j=1,2, \\
& \frac{\partial^{2} \ell\left(\theta_{11}, \theta_{12}, \theta_{21}, \theta_{22} \mid \underline{t}\right)}{\partial \theta_{i j} \partial \theta_{k l}}=0, \quad \text { For each } i j \neq k l .
\end{aligned}
$$

Taking the minus expectation of equations (18)-(20), we readily obtain the Fisher information matrix of the parameters $\theta_{11}, \theta_{12}, \theta_{21}$, and $\theta_{22}$, say $\lambda\left(\theta_{11}, \theta_{12}, \theta_{21}, \theta_{22}\right)$, where

$$
\lambda\left(\theta_{11}, \theta_{12}, \theta_{21}, \theta_{22}\right)=\left(\begin{array}{cccc}
\frac{n_{11}}{\theta_{11}^{2}} & 0 & 0 & 0 \\
0 & \frac{n_{12}}{\theta_{12}^{2}} & 0 & 0 \\
0 & 0 & \frac{n_{21}}{\theta_{21}^{2}} & 0 \\
0 & 0 & 0 & \frac{n_{22}}{\theta_{22}^{2}}
\end{array}\right)_{\text {at } \rightarrow\left(\hat{\theta}_{11}, \widehat{\theta}_{12}, \widehat{\theta}_{21}, \widehat{\theta}_{22}\right)} .
$$


Then, the inverse Fisher information matrix under nonzero values of the elements of the diagonal elements provides the variance-covariance matrix of the MLEs. Hence, the approximate $(1-2 \alpha) \%$ CIs of the parameters $\theta_{11}, \theta_{12}, \theta_{21}$, and $\theta_{22}$, using the fact that $\left(\theta_{11}, \theta_{12}, \theta_{21}, \theta_{22}\right)$ is approximately normal distribution with mean $\left(\theta_{11}, \theta_{12}, \theta_{21}, \theta_{22}\right)$ and covariance matrix $\lambda^{-1}\left(\theta_{11}, \theta_{12}, \theta_{21}, \theta_{22}\right)$, are established by

$$
\left\{\begin{array}{l}
\widehat{\theta}_{11} \mp z_{\alpha} \frac{\widehat{\theta}_{11}}{\sqrt{n_{11}}}, \\
\widehat{\theta}_{12} \mp z_{\alpha} \frac{\widehat{\theta}_{12}}{\sqrt{n_{12}}}, \\
\widehat{\theta}_{21} \mp z_{\alpha} \frac{\widehat{\theta}_{21}}{\sqrt{n_{21}}}, \\
\widehat{\theta}_{11} \mp z_{\alpha} \frac{\widehat{\theta}_{22}}{\sqrt{n_{22}}},
\end{array}\right.
$$

where $z_{\alpha}$ is the upper $100 \alpha$ - th percentile of standard normal distribution.

\section{Bayesian Estimation}

Here, we discuss the Bayesian analysis of the model parameters based on the Type-I HPC competing risk sample.
Both point estimation and credible interval estimation of the unknown parameter vector $\varphi=\left(\theta_{11}, \theta_{12}, \theta_{21}, \theta_{22}\right)$ are considered. Now, we specify the prior distributions of the parameters. It is desirable that the model parameters are independent such that all prior and posterior densities belong to similar families. These prior choices allow the posterior distribution to be analytically tractable and computationally efficient. A natural choice for the priors of $\left(\varphi_{1}, \varphi_{2}, \varphi_{3}, \varphi_{4}\right)=\left(\theta_{11}, \theta_{12}, \theta_{21}, \theta_{22}\right)$ would be to assume that the four quantities are independent gamma $G\left(a_{i}, b_{i}\right), i=$ $1,2,3,4$ distributions, respectively, with the following densities:

$$
\pi_{i}\left(\varphi_{i}\right)=\frac{b_{i}^{a_{i}}}{\Gamma\left(a_{i}\right)} \varphi_{i}^{a_{i}-1} \exp \left(-b_{i} \varphi_{i}\right), \quad \varphi>0,
$$

where $\varphi_{i}$, and $a_{i}, b_{i}$ are chosen to reflect prior knowledge about $\varphi_{i}, i=1,2,3,4$. The joint prior density of (22) is represented as

$$
\pi(\phi)=\prod_{i=1}^{4} \frac{b_{i}^{a_{i}}}{\Gamma\left(a_{i}\right)} \phi_{i}^{a_{i}-1} \exp \left(-b_{i} \phi_{i}\right) .
$$

By combining the joint prior density (23) and likelihood function (12), we have the joint posterior density $\pi(\varphi \mid t, \omega, \delta)$ as follows:

$$
\begin{aligned}
\pi(\phi \mid \mathbf{t}, \boldsymbol{\omega}, \boldsymbol{\delta}) \propto \frac{b_{1}^{a_{1}} b_{2}^{a_{2}} b_{3}^{a_{3}} b_{4}^{a_{4}}}{\Gamma\left(a_{1}\right) \Gamma\left(a_{2}\right) \Gamma\left(a_{3}\right) \Gamma\left(a_{4}\right)} \theta_{11}^{n_{11}+a_{1}-1} \theta_{12}^{n_{12}+a_{2}-} \theta_{21}^{n_{21}+a_{3}-1} \theta_{22}^{n_{22}+a_{4}-1} \exp \\
\cdot\left\{\begin{array}{c}
-b_{1} \theta_{11}-b_{2} \theta_{12}-b_{3} \theta_{21}-b_{4} \theta_{22}-\left(\theta_{11}+\theta_{12}\right) \sum_{i=1}^{J}\left(R_{1 i}+\omega_{i}\right) t_{i}-\left(\theta_{21}+\theta_{22}\right) \sum_{i=1}^{J}\left(R_{2 i}+\left(1-\omega_{i}\right)\right) t_{i} \\
-\left\{\left(D-m_{1}\right)\left(\theta_{11}+\theta_{12}\right)+\left(N-m_{2}\right)\left(\theta_{21}+\theta_{22}\right)\right\} t^{*}
\end{array}\right\} .
\end{aligned}
$$

Hence, the joint posterior density can be expressed as the product of independent gamma densities as follows:

$$
\pi(\phi \mid t, \omega, \delta) \propto \prod_{i=1}^{4} \phi_{i}^{A_{i}-1} \exp \left(-B_{i} \phi_{i}\right)
$$

where

$$
\begin{aligned}
& A_{1}=n_{11}+a_{1}, \\
& A_{2}=n_{11}+a_{2}, \\
& A_{3}=n_{21}+a_{3}, \\
& A_{4}=n_{22}+a_{4},
\end{aligned}
$$

with

$$
\left\{\begin{array}{l}
B_{1}=b_{1}+\sum_{i=1}^{J}\left(R_{i}+\omega_{i}\right) t_{i}+\left(D-m_{1}\right) t^{*}, \\
B_{2}=b_{2}+\sum_{i=1}^{J}\left(R_{i}+\omega_{i}\right) t_{i}+\left(D-m_{1}\right) t^{*}, \\
B_{3}=b_{3}+\sum_{i=1}^{J}\left(R_{i}+\left(1-\omega_{1}\right)\right) t_{i}+\left(N-m_{2}\right) t^{*}, \\
B_{4}=b_{4}+\sum_{i=1}^{J}\left(R_{i}+\left(1-\omega_{1}\right)\right) t_{i}+\left(N-m_{2}\right) t^{*} .
\end{array}\right.
$$

This turns out that the Bayes estimators can be determined based on a selected loss function. It is well known that the error and loss functions play an important role in Bayesian estimation. One of the most popular symmetric 
TABLE 1: Time to failure of male mice which received a radiation dose at age 5-6 weeks.

\begin{tabular}{|c|c|c|c|c|c|c|c|c|c|c|c|c|}
\hline \multirow{6}{*}{ Group 1} & \multirow{2}{*}{ Thymic lymphoma } & 159 & 189 & 191 & 198 & 200 & 207 & 220 & 235 & 245 & 250 & 256 \\
\hline & & 261 & 265 & 266 & 280 & 343 & 356 & 383 & 403 & 414 & 428 & 432 \\
\hline & \multirow{4}{*}{ Other cases } & 40 & 42 & 51 & 62 & 163 & 179 & 206 & 222 & 228 & 249 & 252 \\
\hline & & 282 & 324 & 333 & 341 & 366 & 385 & 407 & 420 & 431 & 441 & 461 \\
\hline & & 462 & 482 & 517 & 517 & 524 & 564 & 567 & 586 & 619 & 620 & 621 \\
\hline & & 622 & 647 & 651 & 686 & 761 & 763 & & & & & \\
\hline \multirow{7}{*}{ Group 2} & \multirow{3}{*}{ Thymic lymphoma } & 158 & 192 & 193 & 194 & 195 & 202 & 212 & 215 & 229 & 230 & 237 \\
\hline & & 240 & 244 & 247 & 259 & 300 & 301 & 321 & 337 & 415 & 434 & 444 \\
\hline & & 485 & 496 & 529 & 537 & 624 & 707 & 800 & & & & \\
\hline & \multirow{4}{*}{ Other cases } & 136 & 246 & 255 & 376 & 421 & 565 & 616 & 617 & 652 & 655 & 658 \\
\hline & & 660 & 662 & 675 & 681 & 734 & 736 & 737 & 757 & 769 & 777 & 800 \\
\hline & & 807 & 825 & 855 & 857 & 864 & 868 & 870 & 873 & 882 & 895 & 910 \\
\hline & & 934 & 942 & 1015 & 1019 & & & & & & & \\
\hline
\end{tabular}

TABLe 2: The Type-II HPC competing risks sample for radiation dose data with $m=60$.

\begin{tabular}{lcccccccccccccc}
\hline$\omega_{i}$ & 1 & 1 & 1 & 1 & 2 & 2 & 1 & 1 & 1 & 1 & 1 & 2 & 2 \\
$t_{\mathrm{i}}$ & 0.040 & 0.042 & 0.051 & 0.062 & 0.136 & 0.158 & 0.159 & 0.163 & 0.179 & 0.189 & 0.191 & 0.192 & 0.193 & 0.194 \\
$\delta_{\mathrm{i}}$ & 0 & 0 & 0 & 0 & 0 & 1 & 1 & 0 & 0 & 1 & 1 & 1 & 1 \\
\hline$\omega_{\mathrm{i}}$ & 2 & 1 & 1 & 2 & 1 & 1 & 2 & 2 & 1 & 1 & 1 & 2 & 2 \\
$t_{\mathrm{i}}$ & 0.195 & 0.198 & 0.200 & 0.202 & 0.206 & 0.207 & 0.212 & 0.215 & 0.220 & 0.222 & 0.228 & 0.229 & 0.230 & 0.235 \\
$\delta_{\mathrm{i}}$ & 1 & 1 & 1 & 1 & 0 & 1 & 1 & 1 & 1 & 0 & 0 & 1 & 1 & 1 \\
\hline$\omega_{\mathrm{i}}$ & 2 & 2 & 2 & 1 & 2 & 2 & 1 & 1 & 1 & 2 & 1 & 2 & 1 & 1 \\
$t_{\mathrm{i}}$ & 0.237 & 0.240 & 0.244 & 0.245 & 0.246 & 0.247 & 0.249 & 0.250 & 0.252 & 0.255 & 0.256 & 0.259 & 0.261 & 0.265 \\
$\delta_{\mathrm{i}}$ & 1 & 1 & 1 & 1 & 0 & 1 & 0 & 1 & 0 & 0 & 1 & 1 & 1 \\
\hline$\omega_{\mathrm{i}}$ & 1 & 1 & 1 & 2 & 2 & 2 & 1 & 1 & 2 & 1 & 1 & 1 & 1 \\
$t_{\mathrm{i}}$ & 0.266 & 0.280 & 0.282 & 0.300 & 0.301 & 0.321 & 0.324 & 0.333 & 0.337 & 0.341 & 0.343 & 0.356 & 0.366 & 0.376 \\
$\delta_{\mathrm{i}}$ & 1 & 1 & 0 & 1 & 1 & 1 & 0 & 0 & 1 & 0 & 1 & 1 & 0 & 0 \\
\hline
\end{tabular}

TABLE 3: The MLEs and BEs of the parameters and corresponding CIs.

\begin{tabular}{|c|c|c|c|c|c|c|c|c|}
\hline & \multirow{2}{*}{ ML } & \multirow{2}{*}{ SEL } & \multicolumn{2}{|c|}{ LINX } & \multicolumn{2}{|c|}{ GE } & \multirow{2}{*}{$95 \%$ asymptotic CI } & \multirow{2}{*}{ 95\% Bayes CrIs } \\
\hline & & & $c=1.0$ & $c=-1.0$ & $q=2.0$ & $q=-2.0$ & & \\
\hline$\theta_{11}$ & 0.9392 & 0.9392 & 0.9142 & 0.9661 & 0.8559 & 0.9664 & $(0.4927,1.3856)$ & $(0.5984,1.3425)$ \\
\hline$\theta_{12}$ & 0.8839 & 0.8839 & 0.8604 & 0.9093 & 0.8006 & 0.9111 & $(0.4508,1.3170)$ & $(0.5544,1.2760)$ \\
\hline$\theta_{21}$ & 0.8544 & 0.8545 & 0.8357 & 0.8742 & 0.7866 & 0.8766 & $(0.4702,1.2385)$ & $(0.5595,1.2002)$ \\
\hline$\theta_{22}$ & 0.1799 & 0.1799 & 0.1759 & 0.1840 & 0.1102 & 0.2011 & $(0.0036,0.3561)$ & $(0.0614,0.3487)$ \\
\hline
\end{tabular}

loss functions is the squared error (SE) loss function which gives equal weight to overestimation as well as underestimation. In some cases, the use of symmetric loss function may be inappropriate; see, for example, Basu and Ebrahimi [20]. For this reason, asymmetric loss functions can be proposed. One of the well-known asymmetric loss functions is the linear exponential (LINEX) loss function which was introduced by Varian [21]. Another alternative function is the general entropy (GE) loss function (see Ren et al. [22]). For estimating any parameter (say, $\varphi_{i}$ ) by a decision rule $\widehat{\varphi}_{i}$, using the Bayes approach, the following loss functions are considered.

(1) Squared error (SE) loss function is defined by the following function:

$$
g_{1}\left(\varphi_{i}, \widehat{\varphi}_{i}\right)=\left(\widehat{\varphi}_{i}-\varphi_{i}\right)^{2}
$$

Then, the Bayes estimators $\widehat{\varphi}_{i B}$ of $\varphi_{i}$ under SE loss function are given by $\widehat{\varphi}_{i B}=E_{\pi}\left(\varphi_{i} \mid t, \omega, \delta\right)$

(2) Linear exponential (LINEX) loss function is defined by

$$
g_{2}\left(\phi_{i}, \hat{\phi}_{i}\right)=\exp \left\{c\left(\hat{\phi}_{i}-\phi_{i}\right)\right\}-c\left(\hat{\phi}_{i}-\phi_{i}\right)-1, \quad c \neq 0 \text {. }
$$

Then, the Bayes estimators $\widehat{\varphi}_{i B}$ of $\varphi_{i}$ under LINEX loss function are obtained to be

$$
\widehat{\varphi}_{i B}=\frac{-1}{c} \log \left[E_{\pi}\left(\exp \left\{-c \varphi_{i}\right\} \mid t, \omega, \delta\right)\right] .
$$

(3) General entropy (GE) loss functions are defined by

$$
g_{3}\left(\varphi_{i}, \widehat{\varphi}_{i}\right)=\left(\frac{\widehat{\varphi}_{i}}{\varphi_{i}}\right)^{q}-q \log \left[\frac{\widehat{\varphi}_{i}}{\varphi_{i}}\right]-1, \quad q \neq 0 .
$$

Then, the Bayes estimator $\widehat{\varphi}_{i B}$ of $\varphi_{i}$ under GE loss is 
TABLE 4: The average estimates and MSEs when $\varphi=(1.0,0.8,1.5,1.2)$.

\begin{tabular}{|c|c|c|c|c|c|c|c|c|c|c|c|c|c|}
\hline \multirow{2}{*}{$(\mathrm{D}, \mathrm{N}, m, \tau)$} & \multirow{2}{*}{ SC } & \multicolumn{4}{|c|}{ MLE } & \multicolumn{4}{|c|}{ Bayes $\left(P^{0}\right)$} & \multicolumn{4}{|c|}{ Bayes $\left(P^{1}\right)$} \\
\hline & & $\theta_{11}$ & $\theta 12$ & $\theta 21$ & $\theta 22$ & $\theta 11$ & $\theta 12$ & $\theta 21$ & $\theta 22$ & $\theta 11$ & $\theta 12$ & $\theta 21$ & $\theta 22$ \\
\hline \multirow{8}{*}{$(30,30,30,1.2)$} & \multirow{2}{*}{ I } & 1.231 & 1.124 & 1.621 & 1.425 & 1.211 & 1.112 & 1.607 & 1.409 & 1.187 & 0.982 & 1.5872 & 1.314 \\
\hline & & 0.241 & 0.204 & 0.421 & 0.374 & 0.217 & 0.192 & 0.403 & 0.355 & 0.189 & 0.114 & 0.354 & 0.301 \\
\hline & \multirow{2}{*}{ II } & 1.199 & 1.111 & 1.604 & 1.413 & 1.187 & 1.095 & 1.589 & 1.339 & 1.142 & 0.941 & 1.5839 & 1.287 \\
\hline & & 0.192 & 0.152 & 0.372 & 0.327 & 0.178 & 0.151 & 0.349 & 0.304 & 0.141 & 0.097 & 0.304 & 0.251 \\
\hline & \multirow{2}{*}{ III } & 1.216 & 1.107 & 1.614 & 1.413 & 1.202 & 1.103 & 1.593 & 1.401 & 1.168 & 0.971 & 1.586 & 1.303 \\
\hline & & 0.224 & 0.190 & 0.404 & 0.355 & 0.200 & 0.175 & 0.384 & 0.337 & 0.171 & 0.101 & 0.336 & 0.289 \\
\hline & \multirow{2}{*}{ VI } & 1.207 & 1.111 & 1.617 & 1.408 & 1.205 & 1.109 & 1.589 & 1.404 & 1.154 & 0.959 & 1.581 & 1.298 \\
\hline & & 0.213 & 0.197 & 0.401 & 0.342 & 0.192 & 0.164 & 0.379 & 0.331 & 0.166 & 0.109 & 0.328 & 0.281 \\
\hline \multirow{8}{*}{$(30,30,40,1.2)$} & \multirow{2}{*}{ I } & 1.132 & 1.045 & 1.587 & 1.356 & 1.122 & 1.022 & 1.514 & 1.301 & 1.089 & 0.902 & 1.527 & 1.229 \\
\hline & & 0.167 & 0.118 & 0.348 & 0.301 & 0.148 & 0.123 & 0.318 & 0.288 & 0.115 & 0.069 & 0.289 & 0.214 \\
\hline & \multirow{2}{*}{ II } & 1.119 & 1.010 & 1.565 & 1.339 & 1.104 & 1.012 & 1.510 & 1.289 & 1.066 & 0.889 & 1.501 & 1.207 \\
\hline & & 0.114 & 0.085 & 0.301 & 0.251 & 0.100 & 0.080 & 0.279 & 0.242 & 0.089 & 0.054 & 0.239 & 0.179 \\
\hline & \multirow{2}{*}{ III } & 1.119 & 1.039 & 1.581 & 1.344 & 1.118 & 1.017 & 1.507 & 1.303 & 1.081 & 0.897 & 1.519 & 1.221 \\
\hline & & 0.149 & 0.104 & 0.331 & 0.291 & 0.132 & 0.107 & 0.304 & 0.279 & 0.100 & 0.061 & 0.279 & 0.211 \\
\hline & \multirow{2}{*}{ VI } & 1.121 & 1.032 & 1.578 & 1.339 & 1.121 & 1.024 & 1.513 & 1.308 & 1.078 & 0.882 & 1.508 & 1.215 \\
\hline & & 0.138 & 0.101 & 0.324 & 0.284 & 0.119 & 0.104 & 0.308 & 0.266 & 0.094 & 0.063 & 0.281 & 0.198 \\
\hline \multirow{8}{*}{$(50,50,60,1.2)$} & \multirow{2}{*}{ I } & 1.104 & 0.982 & 1.542 & 1.314 & 1.110 & 0.978 & 1.529 & 1.303 & 1.061 & 0.875 & 1.511 & 1.209 \\
\hline & & 0.089 & 0.061 & 0.242 & 0.204 & 0.087 & 0.074 & 0.252 & 0.214 & 0.083 & 0.054 & 0.210 & 0.129 \\
\hline & \multirow{2}{*}{ II } & 1.099 & 0.979 & 1.528 & 1.314 & 1.107 & 0.969 & 1.511 & 1.312 & 1.058 & 0.871 & 1.503 & 1.204 \\
\hline & & 0.072 & 0.054 & 0.209 & 0.187 & 0.082 & 0.069 & 0.225 & 0.182 & 0.071 & 0.052 & 0.207 & 0.114 \\
\hline & \multirow{2}{*}{ III } & 1.111 & 0.979 & 1.533 & 1.310 & 1.112 & 0.971 & 1.524 & 1.311 & 1.058 & 0.871 & 1.514 & 1.212 \\
\hline & & 0.084 & 0.058 & 0.215 & 0.206 & 0.081 & 0.071 & 0.248 & 0.209 & 0.081 & 0.055 & 0.210 & 0.114 \\
\hline & \multirow{2}{*}{ VI } & 1.113 & 0.972 & 1.530 & 1.307 & 1.115 & 0.982 & 1.521 & 1.308 & 1.053 & 0.866 & 1.502 & 1.204 \\
\hline & & 0.081 & 0.054 & 0.212 & 0.208 & 0.077 & 0.075 & 0.242 & 0.203 & 0.075 & 0.051 & 0.207 & 0.105 \\
\hline \multirow{8}{*}{$(30,30,40,2.2)$} & & 1.112 & 1.024 & 1.565 & 1.333 & 1.101 & 1.002 & 1.501 & 1.289 & 1.070 & 0.882 & 1.511 & 1.212 \\
\hline & 1 & 0.145 & 0.101 & 0.322 & 0.282 & 0.125 & 0.102 & 0.300 & 0.265 & 0.101 & 0.048 & 0.265 & 0.191 \\
\hline & & 1.111 & 1.001 & 1.557 & 1.325 & 1.082 & 1.007 & 1.503 & 1.278 & 1.058 & 0.872 & 1.490 & 1.189 \\
\hline & 11 & 0.092 & 0.096 & 0.285 & 0.236 & 0.089 & 0.076 & 0.263 & 0.231 & 0.080 & 0.051 & 0.228 & 0.165 \\
\hline & UI & 1.120 & 1.029 & 1.575 & 1.340 & 1.107 & 1.011 & 1.497 & 1.288 & 1.064 & 0.883 & 1.501 & 1.203 \\
\hline & III & 0.124 & 0.081 & 0.304 & 0.269 & 0.1011 & 0.089 & 0.281 & 0.252 & 0.083 & 0.047 & 0.264 & 0.190 \\
\hline & & 1.105 & 1.021 & 1.549 & 1.327 & 1.109 & 1.001 & 1.495 & 1.289 & 1.056 & 0.854 & 1.289 & 1.203 \\
\hline & $\sqrt{ } 1$ & 0.119 & 0.091 & 0.301 & 0.264 & 0.087 & 0.094 & 0.300 & 0.249 & 0.088 & 0.059 & 0.262 & 0.182 \\
\hline & & 1.089 & 0.975 & 1.529 & 1.301 & 1.103 & 0.964 & 1.514 & 1.285 & 1.045 & 0.865 & 1.503 & 1.201 \\
\hline & 1 & 0.089 & 0.061 & 0.242 & 0.204 & 0.087 & 0.074 & 0.252 & 0.214 & 0.083 & 0.054 & 0.210 & 0.129 \\
\hline & II & 1.081 & 0.968 & 1.519 & 1.303 & 1.102 & 0.948 & 1.501 & 1.304 & 1.051 & 0.859 & 1.510 & 1.201 \\
\hline & 11 & 0.068 & 0.049 & 0.188 & 0.155 & 0.071 & 0.061 & 0.211 & 0.169 & 0.064 & 0.049 & 0.201 & 0.101 \\
\hline$(50,50,60,2.2)$ & & 1.103 & 0.968 & 1.525 & 1.304 & 1.103 & 0.964 & 1.513 & 1.302 & 1.051 & 0.868 & 1.501 & 1.203 \\
\hline & 11 & 0.075 & 0.051 & 0.200 & 0.188 & 0.072 & 0.061 & 0.219 & 0.187 & 0.073 & 0.052 & 0.202 & 0.103 \\
\hline & $\mathrm{VI}$ & 1.109 & 0.965 & 1.524 & 1.302 & 1.104 & 0.977 & 1.514 & 1.303 & 1.044 & 0.858 & 1.492 & 1.203 \\
\hline & $\mathrm{V} 1$ & 0.074 & 0.047 & 0.200 & 0.189 & 0.062 & 0.064 & 0.224 & 0.181 & 0.063 & 0.049 & 0.201 & 0.099 \\
\hline
\end{tabular}

$$
\widehat{\varphi}_{i B}=\left(E_{\pi}\left(\omega_{i}^{-q}\right) \mid t, \omega, \delta\right)^{(-1 / q)}
$$

In our setting, we can express the Bayes estimators (BEs) under the SE, LINEX, and GE loss functions as follows.

(1) Under SE loss function, the BEs of model parameters are given by

$$
\widehat{\varphi}_{i B}=\frac{A_{i}}{B_{i}}
$$

(2) Under the LINEX loss function, the BEs of the model parameters are given by

$$
\widehat{\phi}_{i B}=\frac{-A_{i}}{c} \log \left[\frac{B_{i}}{B_{i}+c}\right] .
$$

(3) Under the GE loss function, the BEs of the model parameters are given by

$$
\widehat{\varphi}_{i B}=\frac{1}{B_{i}}\left[\frac{\Gamma\left(A_{i}-q\right)}{\Gamma\left(A_{i}\right)}\right]^{(-1 / q)} .
$$

Clearly, the joint posterior density in (24) can be written as a product of four independent gamma density functions. Hence, the CrIs of parameters vector $\varphi=\left(\theta_{11}, \theta_{12}, \theta_{21}, \theta_{22}\right)$ depend on the distribution of the pivotal $\Psi_{i}=2 \widehat{\varphi}_{i} B_{i}, \quad i=$ 
TABle 5: The ALs and CPs of 95\% CIs when $\varphi=(1.0,0.8,1.5,1.2)$.

\begin{tabular}{|c|c|c|c|c|c|c|c|c|c|c|c|c|c|}
\hline \multirow{2}{*}{$(\mathrm{D}, \mathrm{N}, m, \tau)$} & \multirow{2}{*}{ SC } & \multicolumn{4}{|c|}{ MLE } & \multicolumn{4}{|c|}{ Bayes $\left(\mathrm{P}^{0}\right)$} & \multicolumn{4}{|c|}{ Bayes $\left(\mathrm{P}^{1}\right)$} \\
\hline & & $\theta 11$ & $\theta 12$ & $\theta 21$ & $\theta 22$ & $\theta 11$ & $\theta 12$ & $\theta 21$ & $\theta 22$ & $\theta 11$ & $\theta 12$ & $\theta 21$ & $\theta 22$ \\
\hline \multirow{8}{*}{$(30,30,30,1.2)$} & \multirow{2}{*}{ I } & 2.315 & 1.842 & 3.214 & 2.741 & 2.297 & 1.815 & 3.189 & 2.721 & 2.124 & 1.642 & 3.088 & 2.524 \\
\hline & & 0.89 & 0.89 & 0.90 & 0.89 & 0.90 & 0.91 & 0.89 & 0.90 & 0.90 & 0.91 & 0.90 & 0.91 \\
\hline & \multirow{2}{*}{ II } & 2.295 & 1.801 & 3.169 & 2.702 & 2.245 & 1.765 & 3.141 & 2.671 & 2.075 & 1.590 & 3.037 & 2.480 \\
\hline & & 0.90 & 0.90 & 0.90 & 0.89 & 0.91 & 0.91 & 0.90 & 0.91 & 0.91 & 0.91 & 0.90 & 0.90 \\
\hline & \multirow{2}{*}{ III } & 2.294 & 1.818 & 3.191 & 2.722 & 2.278 & 1.801 & 3.171 & 2.702 & 2.104 & 1.619 & 3.065 & 2.503 \\
\hline & & 0.89 & 0.90 & 0.90 & 0.89 & 0.90 & 0.91 & 0.90 & 0.90 & 0.90 & 0.91 & 0.91 & 0.91 \\
\hline & \multirow{2}{*}{ VI } & 2.288 & 1.814 & 3.187 & 2.719 & 2.269 & 1.803 & 3.164 & 2.705 & 2.101 & 1.609 & 3.051 & 2.497 \\
\hline & & 0.90 & 0.90 & 0.91 & 0.89 & 0.90 & 0.92 & 0.90 & 0.90 & 0.91 & 0.91 & 0.91 & 0.90 \\
\hline \multirow{8}{*}{$(30,30,40,1.2)$} & \multirow{2}{*}{ I } & 2.266 & 1.779 & 3.141 & 2.671 & 2.214 & 1.738 & 3.114 & 2.642 & 2.044 & 1.562 & 3.011 & 2.454 \\
\hline & & 0.91 & 0.90 & 0.91 & 0.91 & 0.90 & 0.92 & 0.91 & 0.91 & 0.92 & 0.93 & 0.90 & 0.92 \\
\hline & \multirow{2}{*}{ II } & 2.219 & 1.731 & 3.102 & 2.624 & 2.270 & 1.701 & 3.075 & 2.602 & 2.001 & 1.514 & 2.950 & 2.413 \\
\hline & & 0.91 & 0.91 & 0.91 & 0.92 & 0.90 & 0.92 & 0.91 & 0.90 & 0.92 & 0.93 & 0.92 & 0.93 \\
\hline & \multirow{2}{*}{ III } & 2.245 & 1.761 & 3.119 & 2.652 & 2.191 & 1.715 & 3.091 & 2.621 & 2.025 & 1.551 & 3.002 & 2.437 \\
\hline & & 0.90 & 0.91 & 0.91 & 0.92 & 0.91 & 0.92 & 0.91 & 0.93 & 0.92 & 0.93 & 0.91 & 0.92 \\
\hline & \multirow{2}{*}{ VI } & 2.238 & 1.762 & 3.121 & 2.648 & 2.185 & 1.709 & 3.088 & 2.618 & 2.024 & 1.547 & 3.005 & 2.429 \\
\hline & & 0.91 & 0.91 & 0.93 & 0.92 & 0.91 & 0.94 & 0.92 & 0.93 & 0.96 & 0.93 & 0.92 & 0.94 \\
\hline \multirow{8}{*}{$(50,50,60,1.2)$} & \multirow{2}{*}{ I } & 2.191 & 1.703 & 3.075 & 2.591 & 2.242 & 1.671 & 3.048 & 2.570 & 1.970 & 1.475 & 2.919 & 2.385 \\
\hline & & 0.92 & 0.90 & 0.93 & 0.92 & 0.95 & 0.92 & 0.91 & 0.92 & 0.92 & 0.93 & 0.94 & 0.96 \\
\hline & \multirow{2}{*}{ II } & 2.145 & 1.650 & 3.024 & 2.544 & 2.203 & 1.624 & 3.007 & 2.519 & 1.924 & 1.429 & 2.880 & 2.327 \\
\hline & & 0.92 & 0.92 & 0.93 & 0.94 & 0.95 & 0.92 & 0.93 & 0.92 & 0.93 & 0.93 & 0.94 & 0.92 \\
\hline & \multirow{2}{*}{ III } & 2.166 & 1.677 & 3.052 & 2.564 & 2.215 & 1.645 & 3.021 & 2.542 & 1.943 & 1.449 & 2.900 & 2.361 \\
\hline & & 0.93 & 0.92 & 0.93 & 0.92 & 0.94 & 0.92 & 0.90 & 0.92 & 0.92 & 0.94 & 0.94 & 0.94 \\
\hline & \multirow{2}{*}{ VI } & 2.157 & 1.669 & 3.048 & 2.555 & 2.217 & 1.641 & 3.017 & 2.533 & 1.945 & 1.439 & 2.892 & 2.354 \\
\hline & & 0.91 & 0.92 & 0.92 & 0.92 & 0.93 & 0.92 & 0.93 & 0.92 & 0.94 & 0.93 & 0.94 & 0.92 \\
\hline \multirow{8}{*}{$(30,30,40,2.2)$} & & 2.247 & 1.758 & 3.125 & 2.649 & 2.185 & 1.719 & 3.100 & 2.622 & 2.025 & 1.541 & 2.980 & 2.437 \\
\hline & 1 & 0.90 & 0.90 & 0.89 & 0.91 & 0.91 & 0.92 & 0.92 & 0.91 & 0.92 & 0.91 & 0.92 & 0.92 \\
\hline & & 2.202 & 1.714 & 3.083 & 2.607 & 2.251 & 1.682 & 3.055 & 2.570 & 1.983 & 1.489 & 2.931 & 2.390 \\
\hline & 11 & 0.92 & 0.92 & 0.91 & 0.91 & 0.93 & 0.92 & 0.92 & 0.91 & 0.94 & 0.93 & 0.92 & 0.95 \\
\hline & UI & 2.224 & 1.744 & 3.102 & 2.631 & 2.169 & 1.700 & 3.074 & 2.604 & 2.003 & 1.528 & 2.984 & 2.419 \\
\hline & III & 0.92 & 0.92 & 0.93 & 0.93 & 0.90 & 0.94 & 0.96 & 0.93 & 0.92 & 0.95 & 0.91 & 0.94 \\
\hline & $\mathrm{VI}$ & 2.219 & 1.745 & 3.103 & 2.631 & 2.164 & 1.691 & 3.071 & 2.602 & 2.007 & 1.532 & 2.989 & 2.411 \\
\hline & $\sqrt{ } 1$ & 0.92 & 0.94 & 0.93 & 0.90 & 0.91 & 0.94 & 0.92 & 0.93 & 0.94 & 0.92 & 0.93 & 0.93 \\
\hline & & 2.168 & 1.679 & 3.061 & 2.568 & 2.225 & 1.649 & 3.031 & 2.548 & 1.952 & 1.461 & 2.903 & 2.371 \\
\hline & 1 & 0.92 & 0.90 & 0.94 & 0.92 & 0.93 & 0.92 & 0.91 & 0.93 & 0.92 & 0.96 & 0.94 & 0.94 \\
\hline & & 2.127 & 1.629 & 3.007 & 2.526 & 2.182 & 1.607 & 2.984 & 2.503 & 1.901 & 1.408 & 2.859 & 2.301 \\
\hline & II & 0.91 & 0.94 & 0.93 & 0.92 & 0.95 & 0.94 & 0.95 & 0.92 & 0.95 & 0.93 & 0.95 & 0.94 \\
\hline$(50,50,60,2.2)$ & & 2.142 & 1.661 & 3.034 & 2.541 & 2.201 & 1.628 & 3.005 & 2.519 & 1.927 & 1.431 & 2.882 & 2.339 \\
\hline & 11 & 0.92 & 0.92 & 0.94 & 0.92 & 0.94 & 0.92 & 0.96 & 0.92 & 0.97 & 0.94 & 0.93 & 0.93 \\
\hline & VI & 2.138 & 1.651 & 3.037 & 2.541 & 2.208 & 1.628 & 3.008 & 2.516 & 1.919 & 1.424 & 2.881 & 2.338 \\
\hline & VI & 0.94 & 0.92 & 0.92 & 0.92 & 0.92 & 0.92 & 0.93 & 0.92 & 0.95 & 0.93 & 0.94 & 0.93 \\
\hline
\end{tabular}

$1,2,3,4$. The values $\Psi_{i}$ are greater than zero and have $\chi^{2}$ distribution with $2 A_{i}$ degrees of freedom (see Kundu and Joarder [19]). Then, $(1-2 \alpha) \%$ CrIs of the parameters vector $(\varphi)$ are given by

$$
\left(\frac{\chi_{2 A_{i}, \alpha}^{2}}{2 B_{i}}, \frac{\chi_{2 A_{i}, 1-\alpha}^{2}}{2 B_{i}}\right), \quad i=1,2,3,4
$$

\section{Data Analysis and Simulation Study}

Now, we perform an analysis of a real data set for illustrative purposes and compare the performances of the different methods of estimation based on Monte Carlo simulations.
5.1. Data Analysis. Let us consider the real data sets given in Hoel [23]. The data given in Table 1 were obtained from a laboratory experiment on two groups of strain male mice which had received a radiation dose of $300 \mathrm{r}$ at an age of 5-6 weeks. The first group of mice (Group-1) lived in a conventional laboratory environment while the second group (Group-2) was in a germ-free environment. Two major causes of death are considered: thymic lymphoma and reticulum cell sarcoma for Cause 1 and Cause 2, respectively. Therefore, we consider that failures of the first group come from the Pop- $\eta_{1}$ and the failures of the second group come from the Pop- $\eta_{2}$. For more convenience, let us divide the data points by 1000 , which will not affect the inference procedures. Under consideration that $m=60, \tau=0.380$, and $\mathbf{R}=(0,0, \ldots, 0,68)$, the jointly Type-I HPCS competing risks random sample is presented in Table 2. The data reported in Table 2 show that 
TABLE 6: The average estimates and MSEs when $\varphi=(0.5,0.3,0.6,0.2)$.

\begin{tabular}{|c|c|c|c|c|c|c|c|c|c|c|c|c|c|}
\hline \multirow{2}{*}{$(\mathrm{D}, \mathrm{N}, m, \tau)$} & \multirow{2}{*}{ SC } & \multicolumn{4}{|c|}{ MLE } & \multicolumn{4}{|c|}{ Bayes $\left(\mathrm{P}^{0}\right)$} & \multicolumn{4}{|c|}{ Bayes $\left(\mathrm{P}^{1}\right)$} \\
\hline & & $\theta 11$ & $\theta 12$ & $\theta 21$ & $\theta 22$ & $\theta 11$ & $\theta 12$ & $\theta 21$ & $\theta 22$ & $\theta 11$ & $\theta 12$ & $\theta 21$ & $\theta 22$ \\
\hline \multirow{8}{*}{$(30,30,30,2.2)$} & \multirow{2}{*}{ I } & 0.624 & 0.412 & 0.724 & 0.325 & 0.611 & 0.403 & 0.713 & 0.314 & 0.584 & 0.375 & 0.682 & 0.275 \\
\hline & & 0.243 & 0.175 & 0.287 & 0.117 & 0.228 & 0.169 & 0.278 & 0.112 & 0.150 & 0.102 & 0.212 & 0.098 \\
\hline & \multirow{2}{*}{ II } & 0.609 & 0.402 & 0.708 & 0.311 & 0.602 & 0.392 & 0.704 & 0.300 & 0.571 & 0.360 & 0.669 & 0.264 \\
\hline & & 0.215 & 0.151 & 0.254 & 0.089 & 0.202 & 0.141 & 0.251 & 0.091 & 0.124 & 0.088 & 0.183 & 0.085 \\
\hline & \multirow{2}{*}{ III } & 0.614 & 0.409 & 0.717 & 0.315 & 0.607 & 0.395 & 0.700 & 0.302 & 0.572 & 0.371 & 0.669 & 0.268 \\
\hline & & 0.229 & 0.162 & 0.271 & 0.108 & 0.217 & 0.154 & 0.268 & 0.103 & 0.138 & 0.091 & 0.200 & 0.096 \\
\hline & \multirow{2}{*}{ VI } & 0.617 & 0.402 & 0.708 & 0.307 & 0.601 & 0.396 & 0.701 & 0.294 & 0.564 & 0.361 & 0.658 & 0.254 \\
\hline & & 0.224 & 0.155 & 0.263 & 0.101 & 0.209 & 0.148 & 0.261 & 0.097 & 0.131 & 0.084 & 0.189 & 0.097 \\
\hline \multirow{8}{*}{$(30,30,40,2.2)$} & \multirow{2}{*}{ I } & 0.589 & 0.389 & 0.691 & 0.288 & 0.581 & 0.378 & 0.684 & 0.278 & 0.562 & 0.348 & 0.651 & 0.250 \\
\hline & & 0.195 & 0.139 & 0.238 & 0.074 & 0.189 & 0.125 & 0.235 & 0.078 & 0.109 & 0.072 & 0.169 & 0.073 \\
\hline & \multirow{2}{*}{ II } & 0.578 & 0.372 & 0.682 & 0.269 & 0.564 & 0.360 & 0.675 & 0.261 & 0.549 & 0.369 & 0.667 & 0.235 \\
\hline & & 0.166 & 0.112 & 0.209 & 0.051 & 0.152 & 0.101 & 0.207 & 0.051 & 0.089 & 0.041 & 0.136 & 0.045 \\
\hline & \multirow{2}{*}{ III } & 0.581 & 0.375 & 0.673 & 0.273 & 0.569 & 0.363 & 0.665 & 0.263 & 0.551 & 0.341 & 0.635 & 0.241 \\
\hline & & 0.182 & 0.125 & 0.222 & 0.059 & 0.174 & 0.112 & 0.219 & 0.066 & 0.100 & 0.058 & 0.152 & 0.060 \\
\hline & \multirow{2}{*}{ VI } & 0.577 & 0.378 & 0.678 & 0.269 & 0.558 & 0.360 & 0.657 & 0.267 & 0.554 & 0.332 & 0.628 & 0.235 \\
\hline & & 0.174 & 0.117 & 0.215 & 0.057 & 0.163 & 0.104 & 0.215 & 0.054 & 0.091 & 0.053 & 0.148 & 0.049 \\
\hline \multirow{8}{*}{$(50,50,60,2.2)$} & \multirow{2}{*}{ I } & 0.561 & 0.349 & 0.663 & 0.251 & 0.541 & 0.338 & 0.657 & 0.239 & 0.531 & 0.344 & 0.641 & 0.219 \\
\hline & & 0.131 & 0.091 & 0.187 & 0.042 & 0.129 & 0.080 & 0.177 & 0.040 & 0.069 & 0.035 & 0.118 & 0.028 \\
\hline & \multirow{2}{*}{ II } & 0.547 & 0.335 & 0.648 & 0.233 & 0.527 & 0.322 & 0.643 & 0.224 & 0.520 & 0.331 & 0.628 & 0.203 \\
\hline & & 0.114 & 0.086 & 0.169 & 0.028 & 0.114 & 0.064 & 0.160 & 0.027 & 0.044 & 0.024 & 0.103 & 0.015 \\
\hline & \multirow{2}{*}{ III } & 0.555 & 0.342 & 0.654 & 0.248 & 0.539 & 0.332 & 0.648 & 0.228 & 0.524 & 0.337 & 0.632 & 0.212 \\
\hline & & 0.125 & 0.084 & 0.182 & 0.037 & 0.115 & 0.077 & 0.164 & 0.028 & 0.051 & 0.024 & 0.110 & 0.021 \\
\hline & \multirow{2}{*}{ VI } & 0.557 & 0.338 & 0.658 & 0.233 & 0.531 & 0.324 & 0.641 & 0.219 & 0.515 & 0.328 & 0.627 & 0.207 \\
\hline & & 0.119 & 0.079 & 0.175 & 0.029 & 0.107 & 0.064 & 0.148 & 0.019 & 0.043 & 0.019 & 0.100 & 0.013 \\
\hline \multirow{8}{*}{$(30,30,40,3.2)$} & & 0.578 & 0.374 & 0.683 & 0.274 & 0.570 & 0.369 & 0.671 & 0.265 & 0.549 & 0.337 & 0.639 & 0.241 \\
\hline & 1 & 0.178 & 0.121 & 0.219 & 0.057 & 0.171 & 0.108 & 0.214 & 0.060 & 0.089 & 0.068 & 0.145 & 0.059 \\
\hline & & 0.569 & 0.361 & 0.671 & 0.256 & 0.551 & 0.347 & 0.663 & 0.248 & 0.537 & 0.356 & 0.658 & 0.222 \\
\hline & 11 & 0.145 & 0.094 & 0.191 & 0.067 & 0.135 & 0.087 & 0.193 & 0.038 & 0.071 & 0.029 & 0.118 & 0.027 \\
\hline & UI & 0.572 & 0.366 & 0.662 & 0.261 & 0.558 & 0.358 & 0.654 & 0.254 & 0.547 & 0.339 & 0.624 & 0.230 \\
\hline & III & 0.164 & 0.108 & 0.204 & 0.047 & 0.156 & 0.100 & 0.204 & 0.052 & 0.085 & 0.042 & 0.138 & 0.045 \\
\hline & $\mathrm{VI}$ & 0.569 & 0.365 & 0.664 & 0.251 & 0.551 & 0.348 & 0.645 & 0.256 & 0.543 & 0.321 & 0.619 & 0.222 \\
\hline & $\sqrt{1}$ & 0.157 & 0.104 & 0.201 & 0.045 & 0.148 & 0.088 & 0.195 & 0.042 & 0.080 & 0.041 & 0.129 & 0.031 \\
\hline & & 0.549 & 0.338 & 0.654 & 0.239 & 0.528 & 0.325 & 0.641 & 0.230 & 0.519 & 0.332 & 0.635 & 0.208 \\
\hline & 1 & 0.114 & 0.074 & 0.168 & 0.027 & 0.109 & 0.064 & 0.161 & 0.029 & 0.045 & 0.022 & 0.102 & 0.007 \\
\hline & UI & 0.527 & 0.322 & 0.639 & 0.227 & 0.518 & 0.314 & 0.629 & 0.215 & 0.508 & 0.324 & 0.617 & 0.192 \\
\hline & 11 & 0.091 & 0.069 & 0.151 & 0.014 & 0.100 & 0.049 & 0.143 & 0.015 & 0.025 & 0.014 & 0.082 & 0.011 \\
\hline$(50,50,60,3.2)$ & & 0.546 & 0.337 & 0.651 & 0.240 & 0.532 & 0.324 & 0.628 & 0.221 & 0.518 & 0.331 & 0.627 & 0.204 \\
\hline & 11 & 0.109 & 0.071 & 0.161 & 0.019 & 0.100 & 0.061 & 0.148 & 0.015 & 0.039 & 0.015 & 0.095 & 0.015 \\
\hline & & 0.551 & 0.331 & 0.645 & 0.228 & 0.524 & 0.315 & 0.637 & 0.213 & 0.508 & 0.317 & 0.621 & 0.192 \\
\hline & $\mathrm{V} 1$ & 0.102 & 0.062 & 0.169 & 0.014 & 0.89 & 0.049 & 0.137 & 0.012 & 0.031 & 0.012 & 0.084 & 0.009 \\
\hline
\end{tabular}

$J=56,\left(m_{1}, m_{2}\right)=(33,23) \quad$ and $\quad\left(n_{11}, n_{12}, \quad n_{21}, n_{22}\right)=$ $(17,16,19,4)$. The random combined sample is selected from both populations, Pop- $\eta_{1}$ and Pop- $\eta_{2}$, of sizes $D=61$ and $N=67$, respectively. For Bayesian analysis, since we do not have any prior information about the model parameters, we consider the noninformative prior by setting $a_{i}=b_{i}=0.0001$, $i=1,2,3,4$. Hence, the MLEs and BEs under SE, LINEX, and GE losses and the corresponding CIs are computed and presented in Table 3. It can be observed from Table 3 that all point estimates are lying within the $95 \%$ asymptotic CIs and Bayes CrIs.

5.2. Simulation Study. Now, we compare the performances of the different methods of estimation based on Monte Carlo simulations. We compare the performances of the MLEs and
BEs in terms of biases and mean square errors (MSEs). In this simulation, two sets of parameter values, $\varphi=(1.0,0.8,1.5,1.2)$ and $(0.5,0.3,0.6,0.2)$, are considered. Here, we consider different effective sample sizes $m=30,40,60$ and different censoring schemes. In this study, we check how the effect of changing the sample size $D+N, m$ censoring scheme $\mathbf{R}$ and the ideal test time $\tau$ is. Under the noninformative and informative priors, the biases and MSEs of the estimates over 1000 replications are computed for various censoring schemes. The algorithm used for the numerical computations can be described as follows:

(1) For given prior information $\left\{\left(a_{i}, b_{i}\right)\right\}_{i=1,2,3,4}$, the true value of the parameter vector $\varphi=\left(\theta_{11}, \theta_{12}, \theta_{21}, \theta_{22}\right)$ is selected almost to satisfy $\varphi_{i}=E\left(\varphi_{i}\right)=a_{i} / b_{i}$. 
TABle 7: The ALs and CPs of $95 \%$ CIs when $\varphi=(0.5,0.3,0.6,0.2)$.

\begin{tabular}{|c|c|c|c|c|c|c|c|c|c|c|c|c|c|}
\hline \multirow{2}{*}{$(\mathrm{D}, \mathrm{N}, m, \tau)$} & \multirow{2}{*}{ SC } & \multicolumn{4}{|c|}{ MLE } & \multicolumn{4}{|c|}{ Bayes $\left(P^{0}\right)$} & \multicolumn{4}{|c|}{ Bayes $\left(P^{1}\right)$} \\
\hline & & $\theta 11$ & $\theta 12$ & $\theta 21$ & $\theta 22$ & $\theta 11$ & $\theta 12$ & $\theta 21$ & $\theta 22$ & $\theta 11$ & $\theta 12$ & $\theta 21$ & $\theta 22$ \\
\hline \multirow{8}{*}{$(30,30,30,2.2)$} & \multirow{2}{*}{ I } & 1.224 & 0.741 & 1.421 & 0.521 & 1.209 & 0.725 & 1.414 & 0.507 & 1.124 & 0.624 & 1.325 & 0.442 \\
\hline & & 0.88 & 0.89 & 0.89 & 0.88 & 0.89 & 0.90 & 0.89 & 0.90 & 0.90 & 0.91 & 0.90 & 0.91 \\
\hline & \multirow{2}{*}{ II } & 1.175 & 0.701 & 1.282 & 0.479 & 1.165 & 0.627 & 1.381 & 0.461 & 1.082 & 0.674 & 1.290 & 0.403 \\
\hline & & 0.90 & 0.89 & 0.90 & 0.90 & 0.91 & 0.92 & 0.90 & 0.91 & 0.90 & 0.91 & 0.94 & 0.92 \\
\hline & \multirow{2}{*}{ III } & 1.204 & 0.727 & 1.409 & 0.504 & 1.189 & 0.711 & 1.400 & 0.492 & 1.111 & 0.605 & 1.309 & 0.427 \\
\hline & & 0.89 & 0.89 & 0.90 & 0.90 & 0.89 & 0.90 & 0.90 & 0.90 & 0.90 & 0.91 & 0.92 & 0.90 \\
\hline & \multirow{2}{*}{ VI } & 1.211 & 0.718 & 1.402 & 0.508 & 1.177 & 0.703 & 1.392 & 0.488 & 1.104 & 0.607 & 1.313 & 0.4189 \\
\hline & & 0.90 & 0.89 & 0.91 & 0.89 & 0.89 & 0.91 & 0.90 & 0.90 & 0.92 & 0.91 & 0.92 & 0.91 \\
\hline \multirow{8}{*}{$(30,30,40,2.2)$} & \multirow{2}{*}{ I } & 1.151 & 0.672 & 1.256 & 0.454 & 1.141 & 0.602 & 1.356 & 0.434 & 1.057 & 0.651 & 1.266 & 0.377 \\
\hline & & 0.91 & 0.90 & 0.90 & 0.90 & 0.91 & 0.93 & 0.92 & 0.91 & 0.92 & 0.91 & 0.93 & 0.93 \\
\hline & \multirow{2}{*}{ II } & 1.114 & 0.635 & 1.218 & 0.415 & 1.107 & 0.561 & 1.321 & 0.401 & 1.021 & 0.612 & 1.228 & 0.331 \\
\hline & & 0.92 & 0.91 & 0.90 & 0.93 & 0.91 & 0.92 & 0.92 & 0.95 & 0.92 & 0.90 & 0.94 & 0.91 \\
\hline & \multirow{2}{*}{ III } & 1.133 & 0.655 & 1.238 & 0.437 & 1.124 & 0.589 & 1.339 & 0.418 & 1.041 & 0.634 & 1.245 & 0.361 \\
\hline & & 0.92 & 0.91 & 0.90 & 0.90 & 0.92 & 0.93 & 0.94 & 0.91 & 0.96 & 0.92 & 0.93 & 0.91 \\
\hline & \multirow{2}{*}{ VI } & 1.138 & 0.649 & 1.227 & 0.428 & 1.119 & 0.591 & 1.328 & 0.409 & 1.044 & 0.627 & 1.239 & 0.365 \\
\hline & & 0.90 & 0.91 & 0.90 & 0.91 & 0.92 & 0.93 & 0.92 & 0.91 & 0.96 & 0.90 & 0.93 & 0.93 \\
\hline \multirow{8}{*}{$(50,50,60,2.2)$} & \multirow[b]{2}{*}{ I } & 1.085 & 0.611 & 1.192 & 0.389 & 1.082 & 0.535 & 1.300 & 0.375 & 1.000 & 0.587 & 1.204 & 0.307 \\
\hline & & 0.93 & 0.92 & 0.90 & 0.93 & 0.93 & 0.92 & 0.94 & 0.93 & 0.93 & 0.90 & 0.94 & 0.93 \\
\hline & \multirow{2}{*}{ II } & 1.052 & 0.577 & 1.156 & 0.353 & 1.057 & 0.501 & 1.266 & 0.341 & 0.964 & 0.553 & 1.169 & 0.289 \\
\hline & & 0.93 & 0.94 & 0.94 & 0.93 & 0.93 & 0.92 & 0.93 & 0.93 & 0.97 & 0.92 & 0.94 & 0.94 \\
\hline & \multirow{2}{*}{ III } & 1.071 & 0.592 & 1.174 & 0.371 & 1.064 & 0.518 & 1.282 & 0.359 & 0.984 & 0.571 & 1.186 & 0.289 \\
\hline & & 0.90 & 0.93 & 0.92 & 0.93 & 0.93 & 0.93 & 0.92 & 0.93 & 0.94 & 0.92 & 0.91 & 0.95 \\
\hline & \multirow{2}{*}{ VI } & 1.076 & 0.585 & 1.167 & 0.366 & 1.057 & 0.509 & 1.277 & 0.3489 & 0.979 & 0.564 & 1.179 & 0.291 \\
\hline & & 0.92 & 0.92 & 0.94 & 0.93 & 0.94 & 0.94 & 0.92 & 0.93 & 0.95 & 0.92 & 0.92 & 0.94 \\
\hline \multirow{8}{*}{$(30,30,40,3.2)$} & $\mathrm{J}$ & 1.134 & 0.654 & 1.239 & 0.438 & 1.127 & 0.587 & 1.338 & 0.418 & 1.041 & 0.629 & 1.248 & 0.360 \\
\hline & 1 & 0.90 & 0.91 & 0.90 & 0.94 & 0.91 & 0.93 & 0.92 & 0.93 & 0.92 & 0.92 & 0.93 & 0.93 \\
\hline & & 1.090 & 0.618 & 1.203 & 0.400 & 1.092 & 0.547 & 1.305 & 0.387 & 1.008 & 0.600 & 1.211 & 0.315 \\
\hline & II & 0.94 & 0.90 & 0.90 & 0.93 & 0.91 & 0.92 & 0.92 & 0.95 & 0.92 & 0.90 & 0.94 & 0.91 \\
\hline & H & 1.133 & 0.655 & 1.238 & 0.437 & 1.124 & 0.589 & 1.339 & 0.418 & 1.041 & 0.634 & 1.245 & 0.361 \\
\hline & III & 0.92 & 0.91 & 0.92 & 0.93 & 0.92 & 0.93 & 0.92 & 0.91 & 0.94 & 0.92 & 0.93 & 0.93 \\
\hline & $\mathrm{VI}$ & 1.121 & 0.631 & 1.209 & 0.413 & 1.102 & 0.581 & 1.307 & 0.400 & 1.028 & 0.614 & 1.223 & 0.351 \\
\hline & $\sqrt{1}$ & 0.92 & 0.92 & 0.94 & 0.93 & 0.92 & 0.93 & 0.93 & 0.91 & 0.96 & 0.92 & 0.93 & 0.92 \\
\hline & & 1.071 & 0.592 & 1.179 & 0.374 & 1.066 & 0.519 & 1.266 & 0.361 & 0.985 & 0.569 & 1.185 & 0.292 \\
\hline & 1 & 0.92 & 0.91 & 0.90 & 0.91 & 0.93 & 0.92 & 0.92 & 0.93 & 0.95 & 0.92 & 0.94 & 0.95 \\
\hline & $\mathrm{U}$ & 1.029 & 0.558 & 1.141 & 0.334 & 1.042 & 0.482 & 1.251 & 0.327 & 0.951 & 0.537 & 1.151 & 0.271 \\
\hline & 11 & 0.92 & 0.92 & 0.94 & 0.92 & 0.93 & 0.93 & 0.92 & 0.91 & 0.90 & 0.94 & 0.94 & 0.96 \\
\hline$(50,50,60,3.2)$ & & 1.057 & 0.577 & 1.158 & 0.349 & 1.048 & 0.507 & 1.269 & 0.339 & 0.967 & 0.559 & 1.171 & 0.271 \\
\hline & III & 0.95 & 0.93 & 0.92 & 0.91 & 0.94 & 0.93 & 0.92 & 0.94 & 0.94 & 0.93 & 0.92 & 0.94 \\
\hline & $\mathrm{VI}$ & 1.061 & 0.569 & 1.154 & 0.3498 & 1.038 & 0.492 & 1.262 & 0.3468 & 0.964 & 0.551 & 1.154 & 0.277 \\
\hline & VI & 0.92 & 0.93 & 0.94 & 0.92 & 0.94 & 0.91 & 0.92 & 0.95 & 0.92 & 0.94 & 0.93 & 0.92 \\
\hline
\end{tabular}

(2) For given $\mathbf{R}$, the value $R_{1 i}$ is generated from $R_{i}$ binomial variant with the probability of success being equal to $1 / 2$ and $R_{2 i}=R_{i}-R_{1 i}$.

(3) For given $m$, generate $m_{1}$ from $m$ binomial distribution with probability of success being equal to $1 / 2$. Then, $m_{2}=m-m_{1}$.

(4) Generate Type-I HPCS competing risks samples of size $m_{k}$ from exponential distributions with parameter $\theta_{k 1}+\theta_{k 2}, k=1,2$.

(5) For given $\tau$ and the joint Type-I HPC competing risks sample, determine the joint hybrid progressive Type-I competing risks sample.
(6) From the joint Type-I progressive competing risks, censoring sample determines $m_{1}$ and $m_{2}$.

(7) The discrete random variables $n_{k j}, k, j=1,2$, are generated from binomial distributions with sample sizes $m_{1}$ and $m_{2}$ with the probability of success $\theta_{k 1} /\left(\theta_{k 1}+\theta_{k 2}\right)$ and $\theta_{k 2} /\left(\theta_{k 1}+\theta_{k 2}\right)$, respectively.

(8) The point and interval estimates based on the likelihood and Bayes approaches are computed.

(9) For comparison purposes, the average estimates (AEs) and MSEs are computed. Furthermore, the average lengths (ALs) and coverage probabilities (CPs) are evaluated. 
(10) The simulation study is conducted using censoring schemes (CSs) described as follows:

$$
\mathrm{CS}=\left\{\begin{array}{l}
\text { I: } R_{i}=\{0,0, \ldots, 0, D+N-m\}, \\
\text { II: } R_{i}=\{D+N-m, 0,0, \ldots, 0\}, \\
\text { III: } R_{i}= \begin{cases}0, & i \neq \frac{m+1}{2} \text { and } m \text { odd or } i \neq \frac{m}{2} \text { and } m \text { even, } \\
D+N-m, & i=\frac{m+1}{2} \text { and } m \text { odd or } i=\frac{m}{2} \text { and } m \text { even, }\end{cases} \\
\text { IV: } R_{i}=\left\{\begin{array}{l}
0, \quad i>\frac{D+N-m}{2}, \\
2, \quad i \leq \frac{D+N-m}{2} .
\end{array}\right.
\end{array}\right.
$$

(11) All results of the simulation study are reported in Tables 4-7 for noninformative prior $P^{0}=\left\{\left(a_{i}\right.\right.$, $\left.\left.b_{i}\right)\right\}_{i=1,2,3,4}=\{0.0001,0.0001\}$ and informative prior $P^{1}$.

\section{Conclusions and Remarks}

In this paper, we consider the problem of estimating the unknown parameters based on the joint Type-I HPC sample coming from two exponential distributions under a competing risks model. The likelihood and Bayesian methods are used for estimating the model parameter involved. In the Bayesian context, the Bayes estimates are obtained under different loss error functions. The asymptotic confidence intervals and Bayes credible intervals are also discussed. It is evident from the numerical results that while the MLEs are close to the ones based on the Bayes method under noninformative prior, the Bayes estimators under informative prior perform well when compared to the MLEs and the Bayes estimates under the noninformative prior. It is observed that when the effective sample $m$ increases, the MSEs of the estimates and the ALs of the confidence intervals get to be decreased. Moreover, the MSEs of the point estimates as well as the ALs and CPs of CIs and CrIs improve when $\tau$ gets large. This observation is expected due to the fact that when $\tau$ increases, more information on the data is obtained.

\section{Data Availability}

The data supporting the finding of this study are available within the article.

\section{Conflicts of Interest}

The authors declare that there are no conflicts of interest.

\section{Authors' Contributions}

The original draft preparation was done by G. A., A. D., and S. A.-K., and the paper was reviewed and edited by M. R., A. A., and A. A. All authors have read and agreed to the published version of the manuscript.

\section{Acknowledgments}

This work was funded by the Deanship of Scientific Research (DSR), King Abdulaziz University, Jeddah, Saudi Arabia, under Grant no. KEP-PhD-61-130-38. The authors, therefore, acknowledge with thanks DSR technical and financial support.

\section{References}

[1] U. V. R. Rao, I. R. Savage, and M. Sobel, "Contributions to the theory of rank order statistics: the two-sample censored case," The Annals of Mathematical Statistics, vol. 31, no. 2, pp. 415-426, 1960.

[2] A. P. Basu, "On a generalized savage statistic with applications to life testing," The Annals of Mathematical Statistics, vol. 39, no. 5, pp. 1591-1604, 1968.

[3] R. A. Johnson and K. G. Mehrotra, "Locally most powerful rank tests for the two-sample problem with censored data," The Annals of Mathematical Statistics, vol. 43, no. 3, pp. 823-831, 1972.

[4] K. G. Mehrotra and R. A. Johnson, "Asymptotic sufficiency and asymptotically most powerful tests for the two sample censored situation," Annals of Statistics, vol. 4, pp. 589-596, 1976.

[5] G. K. Bhattacharyya and K. G. Mehrotra, "On testing equality of two exponential distributions under combined type II censoring," Journal of the American Statistical Association, vol. 76, no. 376, pp. 886-894, 1981. 
[6] K. G. Mehrotra and G. K. Bhattacharyya, "Confidence intervals with jointly type-II censored samples from two exponential distributions," Journal of the American Statistical Association, vol. 77, no. 378, pp. 441-446, 1982.

[7] N. Balakrishnan and A. Rasouli, "Exact likelihood inference for two exponential populations under joint type-II censoring," Computational Statistics \& Data Analysis, vol. 52, no. 5, pp. 2725-2738, 2008.

[8] A. Rasouli and N. Balakrishnan, "Exact likelihood inference for two exponential populations under joint progressive typeII censoring," Communications in Statistics-Theory and Methods, vol. 39, no. 12, pp. 2172-2191, 2010.

[9] A. R. Shafay, N. Balakrishnan, and Y. Abdel-Aty, "Bayesian inference based on a jointly type-II censored sample from two exponential populations," Journal of Statistical Computation and Simulation, vol. 84, no. 11, pp. 2427-2440, 2014.

[10] S. Mondal and D. Kundu, "Point and interval estimation of Weibull parameters based on joint progressively censored data," Sankhya B, vol. 81, no. 1, pp. 1-25, 2019.

[11] A. Childs, B. Chandrasekar, N. Balakrishnan, and D. Kundu, "Exact likelihood inference based on type-I and type-II hybrid censored samples from the exponential distribution," Annals of the Institute of Statistical Mathematics, vol. 55, no. 2, pp. 319-330, 2003.

[12] H.-S. Jeong, J.-I. Park, and B.-J. Yum, "Development of r, T hybrid sampling plans for exponential lifetime distributions," Journal of Applied Statistics, vol. 23, no. 6, pp. 601-608, 1996.

[13] D. R. Cox, "The analysis of exponentially distributed life-times with two types of failure," Journal of the Royal Statistical Society: Series B (Methodological), vol. 21, no. 2, pp. 411-421, 1959.

[14] M. J. Crowder, Classical Competing Risks, Chapman and Hall, London, UK, 2001.

[15] N. Balakrishnan and D. Han, "Exact inference for a simple step-stress model with competing risks for failure from exponential distribution under Type-II censoring," Journal of Statistical Planning and Inference, vol. 138, pp. 4172-4186, 2008.

[16] R. A. Bakoban and G. A. Abd-Elmougod, "MCMC in analysis of progressively first failure censored competing risks data for Gompertz model," Journal of Computational and Theoretical Nanoscience, vol. 13, pp. 6662-6670, 2016.

[17] A. Ganguly and D. Kundu, "Analysis of simple step-stress model in presence of competing risks," Journal of Statistical Computation and Simulation, vol. 86, pp. 1989-2006, 2016.

[18] A. Algarni, A. M. Almarashi, G. A. Abd-Elmougod, and Z. A. Abo-Eleneen, "Two compound Rayleigh lifetime distributions in analyses the jointly type-II censoring samples: DSGT2018," Journal of Mathematical Chemistry, vol. 58, pp. 950-966, 2020.

[19] D. Kundu and A. Joarder, "Analysis of type II progressively hybrid censored data," Computational Statistics and Data Analysis, vol. 50, pp. 2509-2528, 2006.

[20] A. P. Basu and N. Ebrahimi, "Bayesian approach to life testing and reliability estimation using asymmetric loss function," Journal of Statistical Planning and Inference, vol. 29, pp. 21-31, 1991.

[21] H. R. Varian, "A Bayesian approach to real estate assessment," in Studies in Bayesian Econometrics and Statistics in Honor of Leonard, J. Savage, S. E. Fienberg, and A. Zellner, Eds., pp. 195-208, North-Holland Publishing Company, Amsterdam, The Netherlands, 1975.

[22] C. Ren, D. Sun, and D. K. Dey, "Bayes and frequentist estimation and prediction for exponential distribution," Journal of Statistical Planning and Inference, vol. 136, pp. 2873-2897, 2006.

[23] D. G. Hoel, "A representation of mortality data by competing risks,” Biometrics, vol. 28, pp. 475-488, 1972. 